Génét. Sél. Evol., 1987, 19 (2), 267-274

\title{
Note
}

\section{Genotype diet interaction in Fayoumi and Rhode Island Red layers and their crosses}

\author{
M. ABOU-EL-KASSEM ABD-EL-LATIF ${ }^{(*)}$, A. BORDAS, P. MERAT \\ I.N.R.A., Centre de Recherches de Jouy-en-Josas, \\ Laboratoire de Génétique factorielle, F 78350 Jouy-en-Josas
}

\begin{abstract}
Summary
Fayoumi and Rhode Island Red (R.I.R.) layers and their two reciprocal crosses were distributed into 2 groups which received different diets in the laying period. The diets had the same calculated energy level and their total protein content differed by less than 1 p. 100, but one of them contained 40 p. 100 barley and the other contained none. With the barley-containing diet, feed consumption, egg mass, egg number and mean egg weight per hen were reduced, but the effects were more marked in the R.I.R. line and one of the reciprocal crosses, with a significant genotype $\times$ diet interaction for egg mass, average clutch length, total feed intake and its residual component.
\end{abstract}

Key words : Hen, Fayoumi, Rhode Island Red, egg production, feed, barley.

\begin{abstract}
Résumé
Interaction génotype-régime alimentaire chez des pondeuses Fayoumi, Rhode Island et leurs croisements
\end{abstract}

Des poules Fayoumi et Rhode Island (R.I.R.) et leurs 2 croisements réciproques ont été répartis en 2 groupes recevant un régime alimentaire différent en période de ponte. Les 2 régimes avaient la même teneur énergétique et un taux protéique différant de moins de 1 p. 100 mais l'un contenait 40 p. 100 d'orge, l'autre n'en contenait pas. En présence de la ration à base d'orge, la consommation alimentaire et la masse d'œufs produite par poule, ainsi que le nombre et le poids moyen des oufs, étaient abaissés, mais les effets étaient plus marqués dans la lignée R.I.R. et dans l'un des croisements réciproques, avec une interaction régime $\times$ type génétique significative pour la masse d'œufs, la longueur moyenne des séries de ponte, la consommation alimentaire totale et sa composante " résiduelle ».

Mots clés : Poule, Fayoumi, Rhode Island, ponte, régime alimentaire, orge.

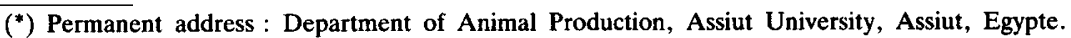




\section{Introduction}

Interactions between genotype and feed composition have generally been recorded in the growth period. Hulan \& Proudfoot (1981) observed interactions between lines and protein level of the ration ; S $\phi$ RENSEN (1980), with 2 lines selected for growth rate with a different protein level, similarly noticed an interaction between lines and protein content of the feed. On the other hand, Hughes (1979) reviewed data on appetite for specific nutrients but did not mention possible genetic or environmental variations for it. The present paper describes an observation possibly related to this field, with a genotype $\times$ feed interaction in Fayoumi and Rhode Island Red hens and their F1 crosses. The initial purpose was to compare these genotypes at 2 protein levels. The actual difference in this respect was lower than expected according to the available components in the rations. On the other hand, the nature of these components differed markedly between the 2 rations. This complicates the interpretation of the results and makes the present work a very preliminary one.

\section{Material and methods}

\section{A. Birds and experimental conditions}

On March 14th and 29 th 1983, two pedigree hatches, from eggs collected over a 3 week period, took place, from 9 Fayoumi and 9 Rhode Island Red (R.I.R.) sires. Each sire was mated to 3 Fayoumi and 3 R.I.R. hens. The R.I.R. line had been bred for a low « residual " feed consumption during the laying period (Bordas \& MERAT, 1984) for 7 generations. The number of sires per generation was 8 , then 9 . This limited number, causing a moderate increase of the inbreeding level, added to the fact that the base population had been kept without selection for 10 generations before the start of the selection experiment, explains why the laying performance was not of a high standard. On the other hand, the Fayoumi line came from pedigree eggs of a line selected for egg number in Cairo University, sent in December 1978. From this time the line was pedigree reproduced in our laboratory without selection, from 8 sires per generation (MÉRAT et al., 1983).

Female chicks, after culling of the smallest families, were raised in floor pens till the age of 17 weeks, then they were put into individual cages in 2 houses (one for each hatch). At the start each house contained 40 pullets of each genetic type (Fayoumi, Fayoumi $\times$ R.I.R., R.I.R. $\times$ Fayoumi, R.I.R.). Pullets from each hatch were randomly distributed into two groups receiving a different feed $(1,2)$ from 17 weeks to the end of the recording period ( 39 weeks of age). The two feeds differed in certain components and (to a limited extent) in their total protein content. Their composition is given in table 1.

\section{B. Measurements}

Egg number was recorded from first egg till the age of 39 weeks. Laying intensity is the ratio (in percent) of egg number to the number of recording days (days from the 
TABLE 1

Composition of the feeds.

\begin{tabular}{|c|c|c|}
\hline Component & $\begin{array}{l}\text { Feed } 1 \\
(\text { p. 100) }\end{array}$ & $\begin{array}{c}\text { Feed } 2 \text { (control) } \\
\text { (p. } 100)\end{array}$ \\
\hline 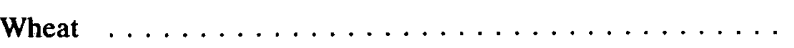 & 10 & 21.5 \\
\hline 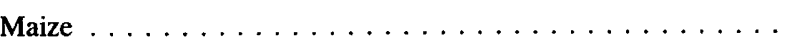 & 26 & 45 \\
\hline 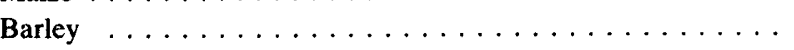 & 40 & - \\
\hline Sunflower meal $\ldots \ldots \ldots \ldots \ldots \ldots \ldots \ldots \ldots \ldots \ldots \ldots$ & 8.6 & 6 \\
\hline Soybean meal $\ldots \ldots \ldots \ldots \ldots \ldots \ldots$ & - & 6 \\
\hline Alfalfa meal $\ldots \ldots \ldots \ldots \ldots \ldots \ldots \ldots$ & - & 6 \\
\hline Meat meal $50 \ldots \ldots \ldots \ldots \ldots \ldots \ldots \ldots$ & 5 & 5 \\
\hline Calcium carbonate $\ldots \ldots \ldots \ldots \ldots \ldots \ldots \ldots \ldots$ & 8 & 8 \\
\hline Mineral and vitamin source $\ldots \ldots \ldots \ldots \ldots$ & 2.5 & 2.5 \\
\hline D.L. méthionine $\ldots \ldots \ldots$ & 0.1 & 0.1 \\
\hline \multicolumn{3}{|l|}{ Analyses } \\
\hline Total proteins (p. 100) & 15.0 & 14.1 \\
\hline Cellulose (p. 100) . . . . . & 4.4 & 4.0 \\
\hline Metabolizable Energy (kcal/kg) (estimated) & 2520 & 2520 \\
\hline Calcium $($ p. 100) $\ldots \ldots \ldots \ldots \ldots \ldots \ldots \ldots \ldots \ldots \ldots$ & 3.5 & 3.5 \\
\hline
\end{tabular}

first egg laid until the age of 39 weeks). A clutch includes eggs laid on successive days. Pauses are defined as periods of at least 2 successive days without an egg ; their total duration is expressed as a percentage of the number of recording days. Mean egg weight was measured between 36 and 38 weeks of age. Finally, over 28 days between the ages of 34 and 38 weeks, 4 traits were measured to estimate feed efficiency :

- mean body weight (W),

- body weight variation during the period $(\Delta \mathrm{W})$,

- total egg mass produced (E),

- feed consumption $(\mathrm{O})$.

The " residual " feed intake $R$, is the deviation of the observed feed intake (O) from that expected from a multiple regression equation on $\mathrm{W}, \Delta \mathrm{W}$ and $\mathrm{E}$ (BYERLY, 1941 ; Gous et al., 1978 ; Mc Donald, 1978 and others).

Feed efficiency is the ratio $\mathrm{O} / \mathrm{E}$.

\section{Statistical analyses}

Hatch, feed and genotype effects and their interactions were tested by 3-way variance analyses taking account of unequal numbers in the subclasses, according to SNEDECOR \& COCHRAN (1957). 


\section{Results and discussion}

Table 2 shows the means for each variable per genotype and feed and the overall means for each feed. Table 3 gives the corresponding variance analyses.

The hatch effects are not relevant here. Moreover, interactions between hatch and genotype have no clear interpretation. However, a possible reason for this is the shortness of the recording period (till 39 weeks of age) with breeds that have a considerable difference of age at the first egg laid; the difference exceeded 8 weeks between the R.I.R. and one of the F1 crosses. The overall genotype effect corresponds to the large and well-known differences in body and eggs size, laying rate and egg shell strength between the Fayoumi and R.I.R. breeds.

Table 2 shows that the crosses were intermediate between the parental breeds for adult body weight and weight gain, mean egg weight, feed consumption. There existed heterosis (the crosses being superior to the midparent), more marked for the Fayoumi $\times$ R.I.R. cross than for the reciprocal, for 17 wk body weight, age at first egg, egg number, laying intensity, mean clutch length, per cent pauses and per cent cracked eggs. For an unknown reason, the laying rate of the R.I.R. line was poorer than expected, even with the control feed. However no sign of disease was recorded.

These results are comparable with those obtained on the same crosses by MÉRAT et al. (1983), showing heterosis for laying rate, egg number and feed efficiency, the Fayoumi $\times$ R.I.R. cross being superior to the reciprocal for egg number. On the other hand, the heterosis in the present comparison on 17 week body weight may be compared with that obtained on earlier growth rate by ABou-EL-KASSEM et al. (1986) on the same parental lines and crosses.

Overall, feed 1 significantly lowered egg mass, laying rate (by 5.6 p. 100) and feed intake (by 4.4 p. 100) and increased the proportion of "pause " days (by 12.9 p. 100). However, these effects of the feeding regime were unequal for the different genetic types. Food intake and egg mass showed a significant genotype $\times$ feed type interaction. For these 2 traits, table 4 shows the means obtained with feed 1 in per cent of those obtained with feed 2 for each genotype.

Although feed intake was relatively less affected than egg mass, the tendency was similar for these 2 variables. Feed 1 reduced them most in the Rhode Island line, then in the R.I.R. $\times$ Fayoumi cross, and the reduction was lower for the Fayoumi and the Fayoumi $\times$ R.I.R. cross. This may be demonstrated in another way by testing the difference between feeds within each genetic type : the t-values are significant only in the R.I.R. line $: \mathrm{t}=2.23(\mathrm{P}<0.05)$ and $\mathrm{t}=2.71(\mathrm{P}<0.01)$ for egg mass and feed intake respectively.

No feed $\times$ hatch nor second order interaction appeared, suggesting that the effect of feed on the different genotypes did not differ markedly in the 2 hatches. In addition, one sees no reason why differences in age at first egg such as that between the R.I.R. breed and the other groups could cause by themselves a different response to a diet given after 17 weeks of age. On the other hand, the 2 reciprocal crosses showed a substantial difference in their response to the 2 feeds and their age at sexual maturity differed little. 


\begin{tabular}{|c|c|c|c|c|c|c|c|c|c|c|c|c|c|c|c|c|c|c|}
\hline & דేత్ & 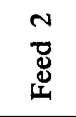 & 品 & 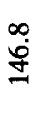 & $\vec{\infty}$ & $\hat{\imath}$ & $\begin{array}{c}\infty \\
\dot{m}\end{array}$ & ڤై & 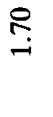 & $\begin{array}{l}0 \\
\dot{o} \\
\dot{f}\end{array}$ & $\begin{array}{l}n \\
\stackrel{n}{0} \\
i \\
-1\end{array}$ & $\begin{array}{l}n \\
0 \\
0 \\
1\end{array}$ & 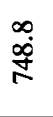 & 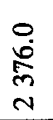 & $\underset{1}{\frac{8}{2}}$ & $\ddot{m}$ & $\stackrel{9}{\dot{\infty}}$ & i্ণ \\
\hline & $\overline{0}$ & 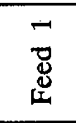 & 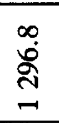 & $\begin{array}{l}\stackrel{\forall}{v} \\
\stackrel{v}{-}\end{array}$ & $\begin{array}{l}\infty \\
\stackrel{\infty}{i}\end{array}$ & $\underset{\mathbb{i}}{\stackrel{\Xi}{*}}$ & $\underset{\text { mi }}{\text { mి }}$ & $\begin{array}{l}8 \\
\text { in }\end{array}$ & $\underset{\sim}{\overrightarrow{0}}$ & $\begin{array}{l}\stackrel{\varphi}{f} \\
\dot{y}\end{array}$ & $\stackrel{\vec{\pi}}{\tilde{n}}$ & 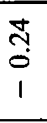 & 宲 & 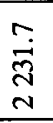 & ๙ู & $\begin{array}{l}\tilde{m} \\
\dot{m}\end{array}$ & ô & 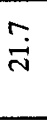 \\
\hline & & $\underset{ن}{~}$ & $\underset{\substack{0\\
}}{\infty}$ & $\begin{array}{l}\infty \\
\infty \\
\infty \\
\infty\end{array}$ & ڤे & $\begin{array}{l}8 \\
8\end{array}$ & $\stackrel{m}{i}$ & $\stackrel{g}{\dot{\Xi}}$ & $\begin{array}{l}F \\
\text { i }\end{array}$ & $\stackrel{\infty}{\stackrel{\infty}{n}}$ & $\begin{array}{l}\stackrel{0}{0} \\
\stackrel{0}{\circ} \\
=\end{array}$ & $\begin{array}{c}q \\
i \\
i\end{array}$ & ఫ্் & $\begin{array}{l}\frac{1}{8} \\
\stackrel{8}{8} \\
\sim\end{array}$ & $\underset{1}{\frac{9}{5}}$ & $\begin{array}{l}\stackrel{8}{\infty} \\
\stackrel{\sim}{1}\end{array}$ & $\ddot{~}$ & $\begin{array}{l}\infty \\
\text { స్ల }\end{array}$ \\
\hline & N & $\begin{array}{l}\dot{2} \vec{े} \\
\dot{x} x\end{array}$ & 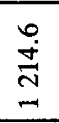 & $\stackrel{m}{\square}$ & $\stackrel{8}{\dot{\&}}$ & $\begin{array}{l}n \\
\stackrel{\infty}{0}\end{array}$ & $\begin{array}{l}\vec{d} \\
\stackrel{\nabla}{*}\end{array}$ & 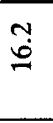 & $\stackrel{+}{+}$ & वें & $\begin{array}{l}\overrightarrow{8} \\
\dot{q}\end{array}$ & 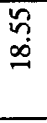 & $\begin{array}{l}\stackrel{+}{\circ} \\
\stackrel{0}{\circ}\end{array}$ & $\begin{array}{l}\infty \\
\stackrel{8}{8} \\
\sim \\
\sim \\
N\end{array}$ & $\frac{9}{2}$ & $\begin{array}{l}8 \\
\text { ले }\end{array}$ & $\stackrel{m}{\infty}$ & $\begin{array}{l}\stackrel{\infty}{\sim} \\
\stackrel{\sim}{*}\end{array}$ \\
\hline & 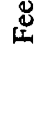 & हن & $\underset{\exists}{ت}$ & $\begin{array}{l}\text { ָे } \\
\underset{\exists}{*}\end{array}$ & ํㅗㅇ & $\stackrel{\sim}{\infty}$ & $\begin{array}{l}\text { fr } \\
\dot{f}\end{array}$ & ڤे & 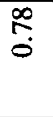 & बें & $\stackrel{0}{\circ}$ & $\underset{\sim}{\widetilde{\sigma}}$ & $\stackrel{m}{\vec{\Phi}}$ & $\stackrel{\vec{\infty}}{\stackrel{\sim}{N}}$ & $\stackrel{\circ}{\stackrel{i}{ }}$ & 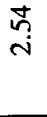 & ڤ̊ & @্ণ \\
\hline కี & & 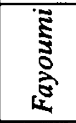 & $\stackrel{\infty}{\stackrel{\infty}{ٍ}}$ & $\stackrel{n}{m}$ & 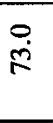 & $\begin{array}{l}\stackrel{+}{T} \\
\stackrel{2}{2}\end{array}$ & ले & 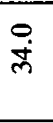 & $\stackrel{\tilde{N}}{0}$ & $\stackrel{\circ}{\infty}$ & $\begin{array}{l}\ddot{0} \\
\ddot{\infty} \\
=\end{array}$ & $\begin{array}{l}+\infty \\
\stackrel{\infty}{9}\end{array}$ & $\begin{array}{l}\ddot{0} \\
\dot{b}\end{array}$ & $\begin{array}{l}0 \\
\stackrel{\tilde{S}}{ } \\
\stackrel{\sim}{N}\end{array}$ & $\frac{a}{\vec{\sigma}}$ & $\underset{+}{\dot{\sigma}}$ & $\stackrel{\sim}{\infty}$ & $\stackrel{a}{a}$ \\
\hline$\stackrel{5}{\stackrel{5}{2}}$ & & $\underset{⿱ 乛 龰}{\dot{z}}$ & $\vec{g}$ & $\begin{array}{l}0 \\
\stackrel{0}{ \pm 0}\end{array}$ & $\stackrel{\circ}{\stackrel{+}{q}}$ & $\frac{a}{i n}$ & $\stackrel{8}{8}$ & ?ุ? & $\begin{array}{l}\stackrel{P}{+} \\
+\end{array}$ & $\frac{r}{\dot{n}}$ & 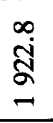 & $\begin{array}{l}80 \\
90 \\
1\end{array}$ & $\frac{n}{n}$ & $\begin{array}{l}\text { @ें } \\
\text { N }\end{array}$ & $\frac{n}{2}$ & $\hat{\mathrm{m}}$ & $\underset{\dot{\infty}}{\infty}$ & $\begin{array}{l}\text { ¿̊. } \\
\text { ¿े }\end{array}$ \\
\hline 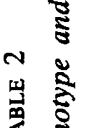 & $\overrightarrow{0}$ & 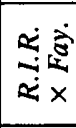 & 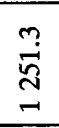 & $\begin{array}{l}\stackrel{\text { I }}{\mathbb{U}} \\
\text { }\end{array}$ & $\underset{\infty}{\dot{\infty}}$ & $\overrightarrow{0}$ & $\underset{\sim}{\stackrel{N}{N}}$ & $\stackrel{\text { I }}{=}$ & ஃ̊ & $\begin{array}{l}\ddot{y} \\
\ddot{f}\end{array}$ & $\begin{array}{l}9 \\
\text { ఫे } \\
\text { ปे }\end{array}$ & $\stackrel{\sim}{\sim}$ & 赵 & $\begin{array}{l}n \\
\tilde{\infty} \\
\tilde{e} \\
\sim\end{array}$ & $\underset{\dot{q}}{\dot{q}}$ & $\begin{array}{l}8 \\
\text { m. }\end{array}$ & $\ddot{\infty}$ & $\begin{array}{l}\stackrel{0}{ } \\
\ddot{n}\end{array}$ \\
\hline 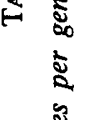 & d & نं & $\stackrel{\sim}{\check{\gamma}}$ & 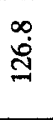 & $\stackrel{n}{\Xi}$ & $\begin{array}{l}0 \\
\ddot{\infty}\end{array}$ & $\begin{array}{l}\text { ga } \\
\dot{+}\end{array}$ & $\stackrel{n}{2}$ & $\stackrel{8}{8}$ & $\tilde{j}$ & $\begin{array}{l}0 \\
\stackrel{a}{a} \\
\sim\end{array}$ & స్ & $\stackrel{\sim}{\infty}$ & $\frac{\vec{i}}{\sqrt{2}}$ & $\begin{array}{l}n \\
8 \\
8\end{array}$ & $\underset{i}{i}$ & $\begin{array}{l}\infty \\
\stackrel{\sim}{r}\end{array}$ & $\begin{array}{l}0 \\
\text { iి }\end{array}$ \\
\hline 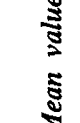 & & 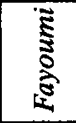 & $\begin{array}{l} \pm \\
\Xi \\
\Xi\end{array}$ & $\stackrel{\sim}{\stackrel{n}{n}}$ & $\stackrel{\sim}{\mathbb{N}}$ & $\hat{i}$ & $\begin{array}{l}\stackrel{\infty}{+} \\
\stackrel{i}{1}\end{array}$ & $\stackrel{\oplus}{m}$ & ถุ? & 品 & $\stackrel{+}{\stackrel{5}{\sigma}}$ & $\stackrel{\widetilde{\sigma}}{\stackrel{\Omega}{=}}$ & $\begin{array}{l}\infty \\
\text { ૂू }\end{array}$ & 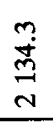 & 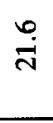 & $\begin{array}{l}\text { ले } \\
\text { ஸे }\end{array}$ & ָ้ & $\overrightarrow{ \pm}$ \\
\hline & & 尊 & 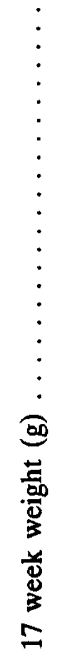 & 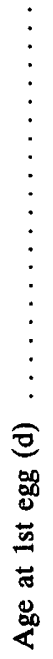 & 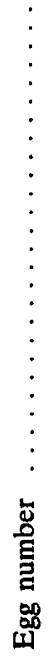 & 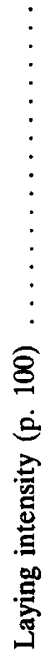 & 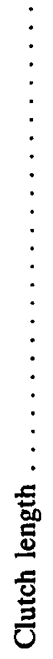 & 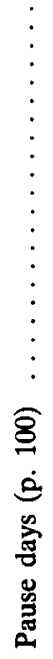 & 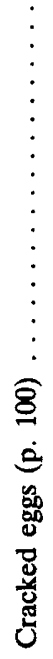 & 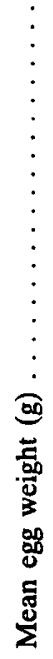 & 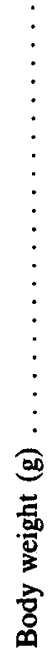 & 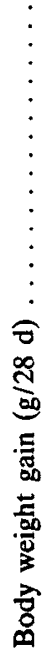 & 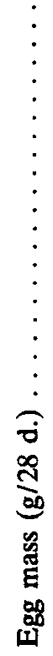 & 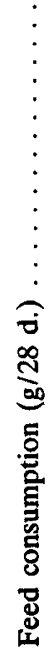 & 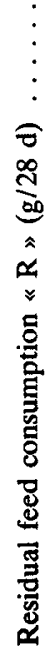 & 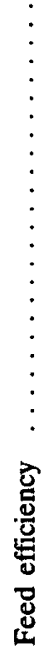 & 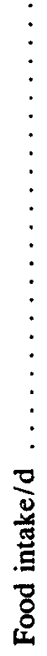 & 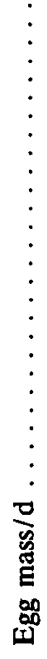 \\
\hline
\end{tabular}




\begin{tabular}{|c|c|c|c|c|c|c|c|c|c|c|c|c|c|c|c|c|}
\hline \multirow{9}{*}{ 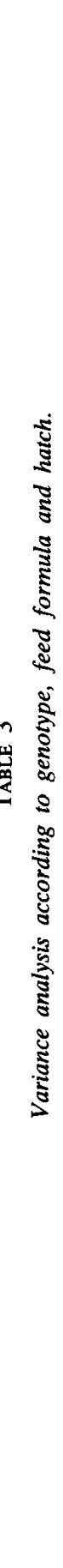 } & & 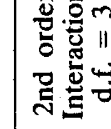 & $\frac{\text { L }}{i}$ & $\overrightarrow{\tilde{o}}$ & $\stackrel{\alpha}{\sigma}$ & $\tilde{n}$ & $\stackrel{8}{2}$ & $\stackrel{v}{0}$ & $\stackrel{\overbrace{}}{\sim}$ & $\stackrel{8}{-}$ & $\stackrel{+}{i}$ & ?̊: & $\stackrel{5}{0}$ & $\stackrel{s}{\sigma}$ & $\stackrel{\rho}{i}$ & \\
\hline & & 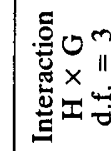 & $\stackrel{\text { fo }}{\stackrel{4}{*}}$ & $\begin{array}{l}\stackrel{\tilde{*}}{*} \\
\stackrel{*}{*} \\
\tilde{\infty} \\
\stackrel{0}{0}\end{array}$ & ஓి & $\begin{array}{l}\tilde{\pi} \\
\widetilde{\sigma} \\
\stackrel{i}{*}\end{array}$ & $\underset{-}{\widetilde{-}}$ & $\begin{array}{l}\stackrel{\tilde{*}}{*} \\
\stackrel{\infty}{\infty} \\
\infty \\
\dot{+}\end{array}$ & 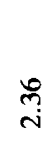 & $\begin{array}{l}0 \\
* \\
* \\
* \\
0 \\
n \\
n \\
n\end{array}$ & $\stackrel{9}{=}$ & 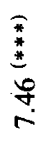 & $\stackrel{\text { q }}{i}$ & $\underset{0}{\tilde{\sigma}}$ & $F$ & \\
\hline & 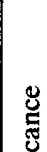 & 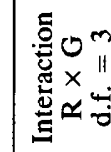 & $\stackrel{\mathbb{D}}{\$}$ & $\stackrel{\text { Tे }}{0}$ & 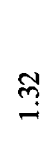 & $\stackrel{2}{=}$ & $\stackrel{\bar{*}}{\tilde{m}}$ & 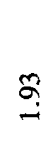 & $\stackrel{m}{0}$ & $\stackrel{\vec{i}}{\vec{i}}$ & $\stackrel{\sim}{\leftrightarrow}$ & ?̊ & $\begin{array}{l}\stackrel{\bar{z}}{\infty} \\
\stackrel{m}{m} \\
\stackrel{m}{m}\end{array}$ & $\begin{array}{l}\stackrel{\tilde{*}}{*} \\
\stackrel{\circ}{n} \\
m\end{array}$ & 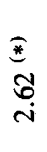 & \\
\hline & 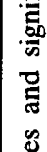 & |l & $\stackrel{+}{0}$ & $\stackrel{R}{i}$ & $\stackrel{\mathscr{2}}{\mathrm{i}}$ & $\stackrel{8}{0}$ & $\overrightarrow{\dot{\sigma}}$ & 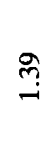 & $\vec{\nabla}$ & $\stackrel{8}{\text { i }}$ & $\stackrel{8}{8}$ & $\stackrel{0}{0}$ & $\stackrel{0}{\stackrel{2}{2}}$ & f̊ & $\underline{\Omega}$ & \\
\hline & $\begin{array}{l}\frac{5}{\pi} \\
\frac{\pi}{2}\end{array}$ & : & $\begin{array}{l}\bar{F} \\
\stackrel{*}{*} \\
\overrightarrow{0} \\
\dot{\infty}\end{array}$ & 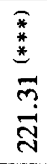 & 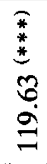 & 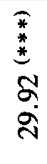 & 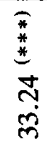 & \begin{tabular}{l}
$\tilde{*}$ \\
$\stackrel{*}{*}$ \\
\multirow{2}{*}{} \\
$\stackrel{\sim}{*}$
\end{tabular} & 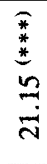 & 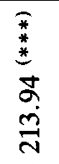 & 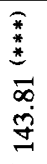 & 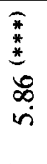 & 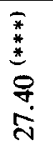 & $\begin{array}{l}\frac{\tilde{F}}{*} \\
\stackrel{*}{*} \\
\sim \\
\stackrel{\sim}{N}\end{array}$ & $\begin{array}{l}\stackrel{\tilde{*}}{*} \\
\stackrel{*}{*} \\
\vec{\sim} \\
0\end{array}$ & \\
\hline & & 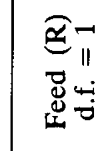 & $\stackrel{8}{8}$ & 苛 & $\underset{m}{8}$ & $\begin{array}{l}\stackrel{*}{*} \\
\stackrel{*}{*} \\
\bar{a} \\
\dot{\sigma}\end{array}$ & 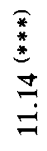 & $\stackrel{n}{i}$ & $\underset{0}{0}$ & 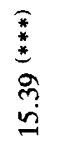 & $\stackrel{\Xi}{0}$ & $\begin{array}{l}8 \\
0\end{array}$ & 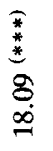 & 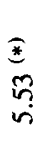 & $\stackrel{a}{0}$ & \\
\hline & & 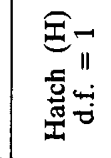 & $\stackrel{\sigma}{0}$ & \begin{tabular}{l}
$\stackrel{\pi}{*}$ \\
$\stackrel{*}{*}$ \\
\multirow{5}{*}{} \\
$\dot{\sim}$
\end{tabular} & กั & $\begin{array}{l}\hat{*} \\
\stackrel{*}{*} \\
\text { बे } \\
\sigma\end{array}$ & 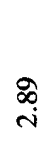 & 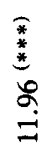 & $\begin{array}{l}\stackrel{F}{*} \\
\underset{+}{*}\end{array}$ & $\begin{array}{l}\hat{*} \\
\stackrel{*}{*} \\
\stackrel{*}{*} \\
8 \\
\stackrel{0}{0}\end{array}$ & 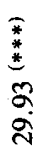 & f & $\underset{n}{\vec{n}}$ & $\stackrel{\check{r}}{\dddot{r}}$ & $\stackrel{m}{m}$ & \\
\hline & & 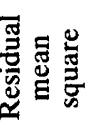 & $\begin{array}{l}\mathscr{O} \\
\infty \\
\infty\end{array}$ & $\stackrel{\sim}{\tilde{\sim}}$ & $\stackrel{n}{q}$ & ڤ్ & $\approx$ & $\stackrel{\mathscr{J}}{\mathrm{N}}$ & $\Xi$ & $\stackrel{\circ}{a}$ & 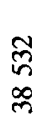 & $\begin{array}{l}\text { J } \\
0\end{array}$ & $\begin{array}{l}\mathscr{D} \\
0 \\
\kappa\end{array}$ & 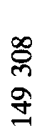 & $\begin{array}{l}\infty \\
\stackrel{\infty}{2} \\
i\end{array}$ & 造 \\
\hline & & $\begin{array}{l}\frac{0}{0} \\
\text { 苞 } \\
\text { > }\end{array}$ & 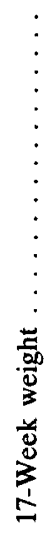 & 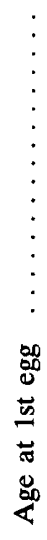 & 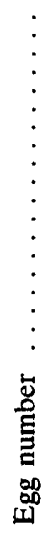 & 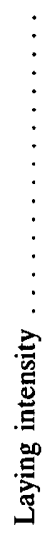 & 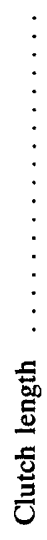 & 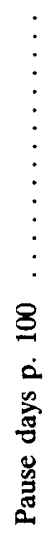 & 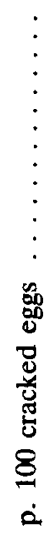 & 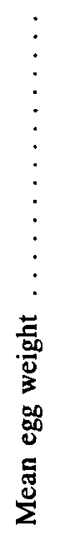 & $\begin{array}{c}\vdots \\
\vdots \\
\vdots \\
\vdots \\
\vdots \\
\vdots \\
\vdots \\
\vdots \\
\frac{5}{0} \\
\frac{1}{0} \\
3 \\
\frac{2}{0} \\
0\end{array}$ & 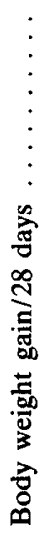 & 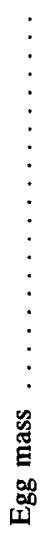 & 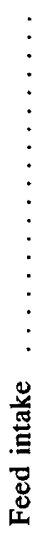 & 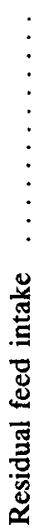 & 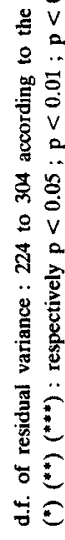 \\
\hline
\end{tabular}


TABLE 4

Mean values obtained by genetic types with the feed 1 in p. 100 of that obtained with the feed 2 for feed intake and egg mass.

\begin{tabular}{l|c|c|c|c}
\hline \multirow{2}{*}{ Variable $(\mathrm{g} / 28$ days $)$} & \multicolumn{3}{|c}{ p. 100 according to genetic type } \\
\cline { 2 - 6 } & Fayoumi & $\begin{array}{c}\text { Fayoumi } \\
\times \text { R.I.R. }\end{array}$ & $\begin{array}{c}\text { R.I.R. } \\
\times \text { Fayoumi }\end{array}$ & R.I.R. \\
\hline Feed intake $\ldots \ldots \ldots \ldots \ldots \ldots \ldots \ldots$ & 96.9 & 99.2 & 95.2 & 86.1 \\
Egg mass $\ldots \ldots \ldots \ldots \ldots \ldots \ldots$ & 88.9 & 93.1 & 81.7 & 62.7 \\
\hline \hline
\end{tabular}

Our data cannot explain unequivocally the unfavorable effects of the experimental feed. The difference between feeds in total protein is rather small. For M.E., the estimated value is identical, and even a moderate difference would not be expected to have large effects on performance. Unfortunately experimental determinations of M.E. were not available. Differences in more specific components like aminoacids cannot be ruled out but they were not determined. Finally, favorable substances might be lacking or substances depressing appetite and/or laying performance might be contained in components specific to the experimental feed. This feed lacked the soybean and alfalfa meal and included less wheat and maize; such changes theoretically should not cause any imbalance. On the other hand, the most important quantitative difference in the composition of the rations is the presence of 40 p. 100 barley in feed 1 ; certain adverse effects associated with this cereal, possibly due to soluble fiber components, have been identified for chicks (Anonymous, 1984). These effects seem to have been found less frequently for laying hens but KARUNAJEEWA \& BAGoT (1977) observed a drop of egg number without decrease of feed consumption with 57 p. 100 barley. GoHL et al. (1978) indicate that in Sweden the use of barley is limited to 250 to $350 \mathrm{~g} / \mathrm{kg}$ in layer diets and showed that the addition on $\beta$-glucanase in barley-containing feeds suppresses the appearance of sticky droppings and poor performance, these problems being due to a viscous factor which is hydrolyzed by $\beta$-glucanase. These studies suggest a possible mechanism through which barley may have had a deleterious effect in feed 1 of the present work.

Our data show that when our Fayoumi and R.I.R. lines are compared the former is less susceptible to the unfavorable factor(s) contained in feed 1 . We pointed that the rate of lay for the R.I.R. breed was rather lower than usual. This might suggest that some disease agent rendered the R.I.R. birds more susceptible to a less favorable diet : this would be an interaction between breed and a combination of environmental factors rather than the feed alone. However, it has already been mentioned that no disease condition was observed.

Although the proportionate reduction was greater for egg production than for feed intake, it is difficult to determine whether the depressing effect on egg production caused the drop in feed consumption, or if the former was a consequence of a lowered appetite for this feed. Possibly the fact that the Rhode Island line has been selected for a low intake with a standard feed may support the latter hypothesis : in other words, 
low consumers of a « normal » feed may be more susceptible to a relatively « unpalatable $»$ feed.

Finally, the difference in the response of the 2 reciprocal crosses is interesting. It may suggest a sex-linked effect, with one of the crosses being closer to its paternal breed.

Received March 4, 1986.

Accepted November 5, 1986.

\section{References}

Abou-El-Kassem Abd-El-Latif M., Bordas A., Mérat P., 1986. Croissance, indice de consommation et composition corporelle de poulets des races Fayoumi, Rhode Island et de leur croisement selon deux taux protéiques de la ration. Génét. Sél. Evol., 18, 213-224.

Anonyme, 1984. L'alimentation des animaux monogastriques. Porc, lapin, volailles. 301 pp., I.N.R.A., Versailles.

Bordas A., Mérat P., 1984. Réponses liées dans une expérience de sélection sur la consommation alimentaire "résiduelle" de coqs et poules Rhode Island. Proc. XVIIth World Poultry Congress, Helsinki, August 8-12, 1984, 106-108. World's Poult. Sci. Assoc., Finnish Branch.

BYERLY T.C., 1941. Feeds and other costs of producing market eggs. University of Maryland. Agric. Exp. Stn. Bull., $\mathrm{n}^{\circ}$ A1.

Gohl B., Aldén S., Elwinger K., Thomke S., 1978. Influence of $\beta$-glucanase on feeding value of barley for poultry and moisture content of excreta. Brit. Poult. Sci., 19, 41-47.

Gous R.M., Byerly T.C., Thomas O.P., Kessler J.W., 1978. A partition equation to predict food and energy intake by laying hens. 16th World's Poultry Congress, Rio de Janeiro, Sept. 17-21, 1978, 2, 1-8. World's Poult. Sci. Assoc.

Hughes B.O., 1979. Appetite for specific nutrients. In : Boorman K.N., Freeman B.M. (ed.), Food intake regulation in poultry, 141-169, British Poultry Science Ltd, Edinburgh.

Hulan H.W., Proudfoot F.G., 1981. The effect of different protein levels in three stage diet system on general performance of chickens reared to rooster weight. Poult. Sci., 60, 172-178.

KarUnajeEwa H., Bagot L., 1977. Effect of litter condition, antibiotics, barley and lucerne meal on egg yolk colour and performance of crossbred layers. Aust. J. Exp. Agric., 17, 926-933.

Mc Donald D., 1978. Feed intake of laying hens. World's Poult. Sci J., 34, 209-221.

Mérat P. Bordas A., L'Hospitalier R., Protais J., Bougon M., 1983. Etude des particularités de la poule Fayoumi. III. Ponte, caractéristiques des oufs, efficacité alimentaire et paramètres physiologiques de poules Fayoumi, Rhode Island et $\mathrm{F}_{1}$ en batteries. Génét. Sél. Evol., 15, $147-166$.

Snedecor G.W., Cochran W.G., 1957. Statistical methods. 5th edition, 593 pp., Iowa State Univ. Press, Ames, Iowa, U.S.A.

S $\phi$ RENSEN P., 1980. Selection for growth rate in broilers fed on diets with different protein level. European Poult. Conf. Hambourg, Sept. 8-12, 1980, 6, 64-71. World's Poult. Sci. Assoc., German Branch. 